\title{
Evaluation of a novel attitude estimation algorithm for a high performance fighter aircraft
}

\author{
Raghavendra P. Kukillaya*1 ${ }^{1}$ Kamali C.*2, Amitabh Saraf**, \\ and Suganya $\mathrm{S} . *$ \\ *Flight Mechanics and Control Division, CSIR-National Aerospace Laboratories, Bangalore, \\ Karnataka, 560 017, India (e-mail: raghavendrapk@nal.res.in ${ }^{1}$, ckamali@nal.res.in ${ }^{2}$ ). \\ **IFCS-National Control Law team, Aeronautical Development Agency, Bangalore,
}

Karnataka, 560 017, India (email: saraf@nal.res.in).

\begin{abstract}
This paper discusses the evaluation of a novel attitude estimation algorithm (Kamali et al. (2013)) for a high performance fighter aircraft. This algorithm employs a new modelling approach in the Extended Kalman Filtering (EKF) framework to estimate aircraft attitude information without using forward acceleration sensor, Global Positioning System (GPS) sensors or magnetometer. Evaluation of the algorithm is conducted using flight data from a high performance fighter aircraft and using flight simulation data. Estimation results during various manoeuvres such as full rolls, inverted loops, split-S manoeuvres, steep climbs, and dives, are studied. Effect of different wind perturbations (gust, shear, turbulence, etc.), on estimation results, is also studied. Results using flight data are compared with those obtained from a GPS-assisted Inertial Navigation System (INS), whereas for results using simulation data, the simulation itself provides true values. Conclusions are drawn for the performance of the algorithm based on simulation and flight data.
\end{abstract}

Keywords: Attitude estimation, evaluation, EKF, air-data, simulation, flight data, wind perturbations, sensor noise, inverted loop, full roll.

\section{NOMENCLATURE}

$\begin{array}{ll}x, y & \text { System state, system output } \\ z & \text { Actual measurements } \\ P & \text { State estimate error covariance matrix } \\ Q & \text { Process noise covariance matrix } \\ R & \text { Measurement noise covariance matrix } \\ u_{i}, v_{i}, w_{i} & \text { Body-frame components of inertial speed } \\ V & \text { Total air-reference speed } \\ u, v, w & \text { Body-frame components of air-reference speed } \\ p, q, r & \text { Aircraft body rotational rates } \\ p_{b}, q_{b}, r_{b} & \text { Rate gyro biases } \\ a_{x}, a_{y}, a_{z} & \text { Aircraft axial, lateral, and normal accelerations } \\ g & \text { Acceleration due to gravity } \\ q_{0}, q_{1}, q_{2}, q_{3} & \text { Aircraft attitude: quaternion angles } \\ \phi, \theta, \psi & \text { Aircraft attitude: Euler angles } \\ \alpha & \text { Angle of attack (AoA) } \\ \beta & \text { Angle of side-slip (AoSS) } \\ \gamma & \text { Flight path angle } \\ h & \text { Geometric altitude } \\ h_{p} & \text { Pressure altitude }\end{array}$

\section{INTRODUCTION}

Typical control laws for modern fighter aircraft require only the following measurements as feedback: $p, q, r$ (rate gyros), $\alpha, \beta, V$ (air-data sensors), and $a_{y}, a_{z}$ (two axes accelerometers). Thus, the Digital Flight Control Computer (DFCC) of such aircraft (including the one discussed in this paper) receives data only from the air-data sensors, three axes gyros, and two axes accelerometers (no $a_{x}$ ). These sensors and the associated processing systems have sufficient redundancy and failure detection logics (both in hardware and software) to provide safety critical functionality.

Currently, requirements for the fighter aircraft under discussion are such that pitch and roll angle measurements are needed in the control laws to provide for gravity compensation during turn manoeuvres. Heading angle is not required. Inertial Navigation System (INS) is the standard equipment that provides attitude information on the aircraft (Crassidis et al. (2007)). But, here the safety-critical control laws of DFCC cannot depend on INS due to the following two reasons. First, redundancy requirements are not met since there is only a single INS. Secondly, since INS signals have to be routed from the avionics system to the DFCC, any failure of communication buses or failures of avionics computers (which are only mission-critical) can jeopardize aircraft flight safety. Hence there is a need to compute the aircraft attitudes within the DFCC using only the highintegrity flight control system sensors.

The structure of most estimation techniques is such that the complete set of three axes accelerometers, three axes rate gyros, and magnetometer/GPS sensors are used for attitude estimation (Crassidis et al. (2007), Jung and Tsiotras (2007), Mahony et al. (2008), Park and How (2004), Titterton and Weston (2004), Wendel and Trommer (2004)). But, the estimation algorithm which is evaluated in this paper employs a modelling technique (Kamali et al. (2013)) in the Extended Kalman Filtering (EKF) framework such that it obtains pitch and roll angle information with reasonable accuracy just by using the sensors directly connected to the 
DFCC: three axes rate gyros $(p, q, r)$, two axes accelerometers $\left(a_{y}, a_{z}\right)$, and air-data sensors $(\alpha, \beta, V, h)$. The novelty of the approach lies in avoiding problems of observability arising while estimating attitudes (pitch and roll) in the absence of $a_{x}$ sensor. The idea is to use inertial altitude as one of the states, since altitude rate also contains terms that use pitch and roll attitudes. Altitude can be directly measured using independent static pressure measurements. Air-data sensors are used instead of GPS to obtain linear velocities. Since magnetometer or GPS signals are not used, observability of heading angle is not guaranteed. However, aircraft heading information is not required by the control laws for improving aircraft performance.

In the current work, the performance of this estimation algorithm is evaluated using simulation and flight data. Simulation data is obtained using the in-house nonlinear 6DOF flight simulation software. In this case, various manoeuvres are simulated in the presence of random noise, sensor biases and different forms of wind perturbations. Aircraft flight data has been collected from various flight tests of a high performance aircraft. Data from a total of 332 flights has been used from start to end for the evaluation of this estimation algorithm. The flights include those that are flown in different wind conditions such as cross-winds and turbulence, and also include those that have extreme manoeuvres such as high-speed dives and high-AoA wind-up turns. As far as estimation performance is considered, the target accuracy that is expected from the algorithm in pitch and roll are within \pm 5 and $\pm 10 \mathrm{deg}$, respectively.

The paper is organized as follows. A brief description of the estimation algorithm is given in next section. For more details, refer Kamali et al. (2013). In Section 3, evaluation procedure is described for both simulation and flight data. Estimation results using simulation data are discussed in detail in Section 4, whereas those using flight data are described in Section 5. The results are summarized in Section 6. Conclusions are given in Section 7.

\section{ESTIMATION ALGORITHM}

\subsection{Problem statement}

Pitch and roll angle measurements are required in DFCC of the high performance fighter aircraft under discussion in this paper. The attitude angles are to be used in the aircraft control laws to provide for compensation of gravity terms during turn manoeuvres. Since the use of attitude information from INS is not a feasible solution, it is required to estimate pitch and roll angles using the sensors directly connected to DFCC: three axes rate gyros, two axes accelerometers, and air-data sensors. Note that aircraft heading is not required by the control laws in DFCC.

\subsection{Modelling procedure}

The aircraft under consideration is a fighter aircraft and hence the attitude formulation is carried out using quaternion. The quaternion parameterization is preferred due to the following reasons: 1) it is free from singularities, i.e., the gimbal lock situation is prevented and 2) the rotation matrix is algebraic in quaternion components, thus avoiding transcendental equations.

The modelling structure of proposed EKF estimator (Kamali et al. (2013)) is such that it estimates quaternion components $\left(q_{0}, q_{1}, q_{2}, q_{3}\right)$, gyro biases $\left(p_{b}, q_{b}, r_{b}\right)$, and aircraft altitude (h) as states. The inputs to the estimator are aircraft angular rates $(p, q, r)$, and aircraft velocity components $(u, v, w)$ constructed from air-data measurements, all taken from flight control-based inertial sensors and air-data sensors. The measured outputs are aircraft body accelerations $\left(a_{y}, a_{z}\right)$, and pressure altitude $\left(h_{p}\right)$.

Consider a dynamic system with process and measurement noise. The system can be represented in state-space mathematical form as follows:

$$
\begin{aligned}
\dot{x}(t) & =f(x(t), u(t))+w(t), \\
y(t) & =h(x(t), u(t)), \\
\text { and } z\left(t_{k}\right) & =y\left(t_{k}\right)+v\left(t_{k}\right) .
\end{aligned}
$$

Here $x(t)$ is the state vector, $u(t)$ is the control vector, $w(t)$ is the process noise, and $y(t)$ is the output. However, for any dynamic system, this output is measured at discrete time intervals. This measurement step is shown in (3), where $z\left(t_{k}\right)$ is the measurement at time instant $\mathrm{t}_{\mathrm{k}}$ and $v\left(t_{k}\right)$ indicates the measurement noise.

For the current system, the dynamic state equations consist of eight first-order ordinary differential equations (ODEs). The rotational kinematic equations in terms of the quaternion states form the first four. Rate of change of altitude is the fifth equation. The last three are the gyro bias equations in which the biases are basically considered to be constant in time. For details, see Kamali et al. (2013). The output equations (measurement model) are defined as given below.

$$
\begin{aligned}
& a_{y}=\dot{v}_{i}-\left(-\left(r-r_{b}\right) u_{i}+\left(p-p_{b}\right) w_{i}+g \sin \phi \cos \theta\right) \\
& a_{z}=\dot{w}_{i}-\left(\left(q-q_{b}\right) u_{i}-\left(p-p_{b}\right) v_{i}+g \cos \phi \cos \theta\right) \\
& h_{p}=h
\end{aligned}
$$

Here $u_{i}, v_{i}, w_{i}$ are the three body components of the aircraft inertial (ground) velocity. Thus, available accelerometer measurements are modelled in terms of linear acceleration components, components of centripetal acceleration due to curved path of the vehicle, and gravity vector.

\subsection{EKF algorithm}

The actual prediction and correction update steps of the proposed EKF estimation algorithm and their implementation procedures are generic, similar to any other, and have been detailed in Kamali et al. (2013). For the analysis using simulation data (including cases with sensor noise, bias and wind perturbations), the values given below are chosen for the tuning parameters (covariance matrices).

Process noise, $Q=\operatorname{diag}\left[10^{-7} 10^{-7} 10^{-7} 10^{-7} 0.10000\right]$ (7)

Measurement noise, $R=\operatorname{diag}\left[\begin{array}{lll}1 & 1 & 10\end{array}\right]$

Initial filter error,

$P_{0}=\operatorname{diag}\left[10^{-7} 10^{-7} 10^{-7} 10^{-7} 10^{-7} 10^{-7} 10^{-7} 10^{-7}\right]$ 
For the estimation analysis using flight data, data was actually obtained from multiple versions (different production series) of the same high performance aircraft. There were a few differences in the estimation results across these different production versions. Nevertheless, a common set of tuning parameters was found which gave good estimation performance for all the production versions. This particular set of covariance matrices is given below.

Process noise, $Q=\operatorname{diag}\left[10^{-7} 10^{-7} 10^{-7} 10^{-7} 11000\right]$

Measurement noise, $R=\operatorname{diag}\left[\begin{array}{lll}1 & 1 & 100\end{array}\right]$

Initial filter error,

$P_{0}=\operatorname{diag}\left[10^{-7} 10^{-7} 10^{-7} 10^{-7} 10^{-7} 10^{-7} 10^{-7} 10^{-7}\right]$

It is clear from (7) to (12) that analysis using simulation and flight data has converged to an almost similar set of tuning parameters. The only difference is in the noise characteristics of pressure altitude. Higher values of $Q(5,5)$ and $R(3,3)$ were required in the flight data cases in order to correct for larger perturbations in altitude (50 to $100 \mathrm{~m}$ ) due to transonic jumps. (A transonic jump is an abrupt and discontinuous change in air-data measurements when aircraft flies through transonic speeds.)

\subsection{Approximations}

Information on inertial velocities is actually required, as seen from (4) and (5). But the only measurements available are the air-reference speeds (from air-data sensors), since there is no other inertial velocity sensor, such as GPS, connected. Thus, there is an approximation in that wind velocities are ignored and air-reference velocity components are used as inertial velocity components in the equations. The rates of change of velocities $\left(\dot{v}_{l}, \dot{w}_{l}\right)$ are obtained by numerical differentiation of these air-reference velocity components ( $v$ and $w)$ using backward differences $(13,14)$.

$\dot{v}_{i}=\left(v\left(t_{k}\right)-v\left(t_{k-1}\right)\right) / \Delta t$

$\dot{w}_{i}=\left(w\left(t_{k}\right)-w\left(t_{k-1}\right)\right) / \Delta t$

Here, $\Delta t=t_{k}-t_{k-1}$ is the sampling time. The novelty of the estimation method comes from the fact that even though DFCC does not have information from $a_{x}$ or GPS sensors, how the estimator still manages to estimate pitch and roll angles without getting into observability problems.

\section{EVALUATION PROCEDURE}

\subsection{Simulation data}

Simulation data contains various manoeuvres such as pull up, full roll, dive, and inverted loop. In order to produce realistic simulations, wind effects, random noise and sensor biases, are also introduced. Wind effects are included in the form of gusts, shear or turbulence. Both fixed and random forms of realistic sensor biases are considered in various sensor measurements (gyros, accelerometers, and air-data sensors). In order to study individual effects of different noisy sensors, a step by step procedure is followed to introduce biases into system sensors. This results in four cases of noisy sensor data as given below.
CASE 1: Clean sensor data; no biases or noise.

CASE 2: Realistic fixed biases and random noise are added only in rate gyro measurements $(p, q, r)$. Fixed components have the following values: $p_{b}=-0.2 \mathrm{deg} / \mathrm{s}, q_{b}=0.1 \mathrm{deg} / \mathrm{s}$, and $r_{b}=0.1 \mathrm{deg} / \mathrm{s}$. The random noise component has a standard deviation $0.1 \mathrm{deg} / \mathrm{s}$.

CASE 3: On top of case 2, fixed bias of $0.5 \mathrm{~m} / \mathrm{s}^{2}$ and random noise with standard deviation $0.1 \mathrm{~m} / \mathrm{s}^{2}$ are added to accelerometer measurements $\left(a_{y}, a_{z}\right)$.

CASE 4: On top of case3, fixed bias with following values: 1 $\mathrm{m} / \mathrm{s}, 0.5 \mathrm{deg}, 0.5 \mathrm{deg}$, and $10 \mathrm{~m}$, and random noise with following standard deviations: $0.5 \mathrm{~m} / \mathrm{s}, 0.05 \mathrm{deg}, 0.05 \mathrm{deg}$, and $0.1 \mathrm{~m}$, are added to the measurements TAS, $\alpha, \beta$, and pressure altitude, respectively.

Evaluation of the estimation algorithm is run as a batch process for each simulation data. The prediction and measurement update steps occur at the rate of $80 \mathrm{~Hz}$. For comparing the estimation results, simulation data itself provides the true values.

\subsection{Flight data}

Aircraft flight data has been collected from various flight tests of a high performance aircraft. In order to ease the process of assimilating the estimation results, the flight tests are also categorized into different classes such as high-speed dives, flights with cross-winds, steep dives, turbulenceaffected flights, flights containing high-AoA wind-up turns, and manoeuvring flights.

Evaluation of the estimation algorithm is done using batch simulations for chosen flight sorties from a time instant before take-off to a time instant after landing that is defined by sufficiently high speed. Estimation is run at the rate of 40 Hz. If a particular parameter in the flight data is available at a lower or different frequency, it is appropriately interpolated to match the frequency of $40 \mathrm{~Hz}$. The correctness of estimation results is verified by comparing it with data from a GPS-assisted INS.

\section{ESTIMATION RESULTS: SIMULATION DATA}

In this section and the next, we look at estimation results for some specific manoeuvres, first using simulation (this section) and subsequently using flight data (Section 5). These specific cases are further studied to better understand the dynamic behaviour of estimates under different manoeuvring conditions and as a function of various system parameters.

In the figures below (except Figs. 1 and 2), estimation results are compared with true data by plotting the state estimate (black dashed, 'est') along with the true value (red solid, 'true') as a function of time. If the particular state (such as $\theta$, $\varphi$, or $h$ ) is denoted by $\mathrm{X}$, then $\Delta \mathrm{X}$ denotes the error between the true value and the estimate $\left(\Delta \mathrm{X}=\mathrm{X}_{\text {true }}-\mathrm{X}_{\text {est }}\right)$. In case of simulation data, true values are from simulation itself, whereas for flight data, INS-GPS data provides the true values.

In subsection 4.1, a specific case is reported from results using simulation data in order to discuss the effect of various 
sensor noises on estimation performance. Later, estimation results for simulated inverted-loop and full-roll manoeuvres are studied in subsections 4.2 and 4.3, respectively.

\subsection{Simulation data}

First, we define the following simulation.

Simulation I: A single-pulse input in pitch stick from $t=60$ sec to $t=62 \mathrm{sec}$ (a pull-up manoeuvre) and a roll-stick doublet command from $t=75 \mathrm{sec}$ to $t=83 \mathrm{sec}$ (a couple of full-roll manoeuvres), under wind-shear conditions (initiated at $t=10 \mathrm{sec}$ ). Wind shear profile is such that wind direction is due east and wind speed varies linearly with altitude starting from $5 \mathrm{~m} / \mathrm{s}$ at ground to $30 \mathrm{~m} / \mathrm{s}$ at $10 \mathrm{~km}$.

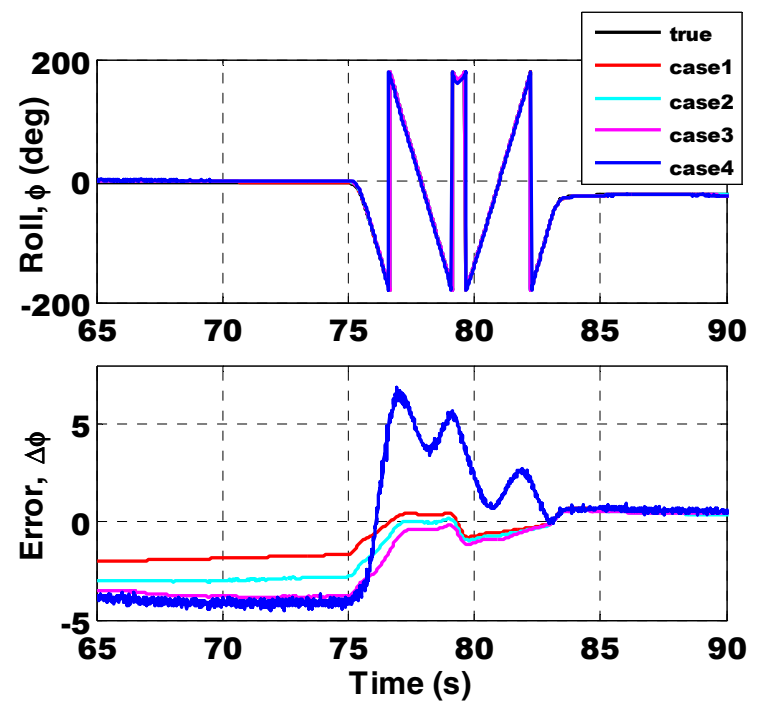

Fig. 1: Comparison of roll estimation results for four cases of noisy simulation data (Simulation I + sensor bias).

In order to get the results of Figs. 1 (roll) and 2 (biases), four sets of noisy simulation data are first generated by applying each of the cases 1 to 4 of noisy sensor data (Subsection 3.1) on Simulation I, and then the estimation algorithm is run for each of the four data sets. Thus, the curve for case 1 (red) shows the estimation results for Simulation I with no sensor bias. (The discrete jumps in biases at $t \approx 10 \mathrm{sec}$ are due to the abrupt initiation of wind shear using a step input.) When fixed and random biases are introduced into the rate gyros (case 2), the estimation performance deteriorates (cyan curve). With sensor bias cases 3 and 4, performance worsens.

Noisy data does not have much effect on convergence of $p_{b}$ (Fig. 2), although convergence does slightly worsen in cases 3 and 4. Convergence behaviour for all biases in case 2 (corrupted rate gyros) is same as in case 1 (Fig. 2), i.e., bias estimates converge to their respective true values (in case 1 , it is zero for all three gyros). But note that, in the presence of wind shear, $r_{b}$ partially loses its observability, i.e., takes a long time to converge (Fig. 2, bottom, cases 1 and 2). In cases 3 and $4, q_{b}$ goes in the right direction, but cannot attain the steady state value (Fig. 2, middle). $r_{b}$ (Fig. 2, bottom) loses its convergence in cases 3 and 4. Especially, it jumps from negative to positive values (and back) during the full- roll manoeuvres. This does not create a problem since $r_{b}$ does not affect pitch or roll but only affects heading angle.

It is clear from Fig. 1 (bottom) that roll estimation errors are relatively much larger in the fourth case, where, in addition to other sensors, noise and bias are introduced into the air-data measurements. Similarly, pitch estimation errors are also relatively larger in the fourth case (not shown). Further, the effects of air-data noise are relatively higher during the fullroll manoeuvres. (Note the bias fluctuations in Fig. 2 during the full-roll manoeuvre.) Even if wind-shear conditions are removed from the simulation, effects of air-data noise remain the same (not shown). These results indicate the importance of air-data measurements in achieving good estimation performance during dynamics manoeuvres.

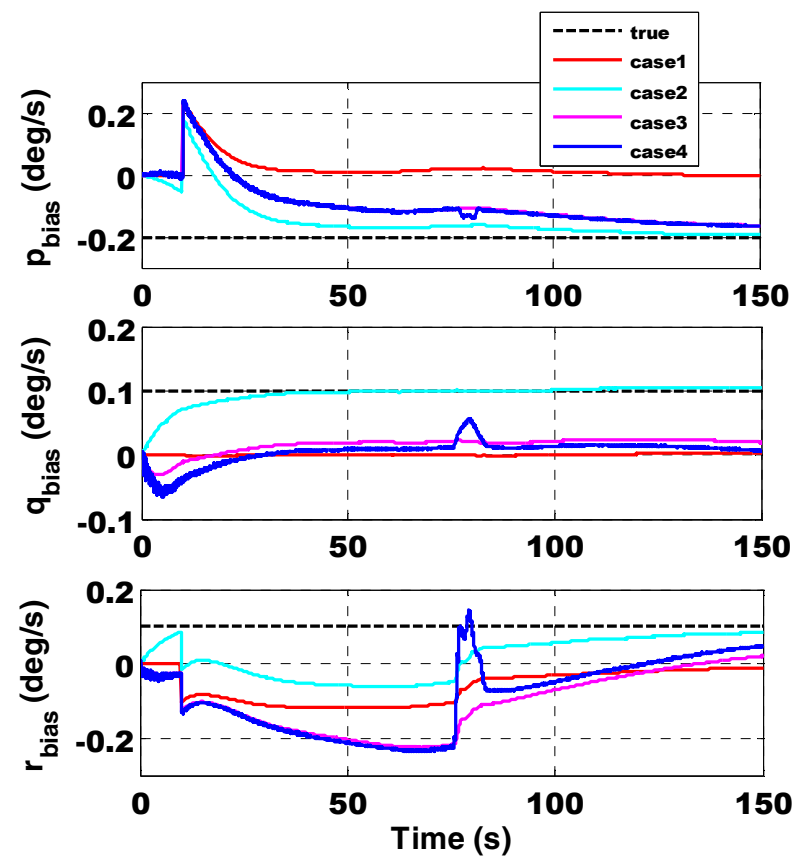

Fig. 2: Time variation of estimated gyro biases for four cases of noisy simulation data (Simulation I + sensor bias). Here the true value of bias (black dashed) is applicable only for cases 2,3 , and 4 .

An accurate measurement model is necessary for good estimation results. During dynamic manoeuvres, the first three terms of $a_{y}$ and $a_{z}$ measurement models (given in (4) and (5)) become important (non-zero body rates and non-zero linear accelerations). These terms entirely depend on the linear velocity measurements, which, in our case, are derived from air-data. Thus, during dynamic manoeuvres, quality of estimation performance depends a lot on accuracy of air-data information. But note that, during manoeuvres in the longitudinal plane, the biases (equivalently, pitch and roll angles (not shown)) are not affected by these noisy air-data measurements (Fig. 2; pull-up at $t=60 \mathrm{sec}$ and push downpull up at $t=85 \mathrm{sec}$ ). The reason for this is that, in these pitch-plane manoeuvres additional reliable information is available through the pressure altitude measurement. (In fullroll manoeuvres, pressure altitude does not vary and hence not much information is available.) 
Table 1 reports the maximum pitch and roll estimation errors for Simulation I. It is noted that the performance is satisfactory for full-roll and pull-up manoeuvres in the presence of wind shear.

Table 1: Estimation errors for Simulation I

\begin{tabular}{|c|c|c|c|c|}
\hline Attitude & CASE 1 & CASE 2 & CASE 3 & CASE 4 \\
\hline$\Delta \phi$ & 5.3216 & 6.3290 & 6.6735 & 6.8171 \\
\hline$\Delta \theta$ & 0.1560 & 0.2190 & 0.4968 & 2.0550 \\
\hline
\end{tabular}

\subsection{Simulation II: inverted loop}

Figure 3 shows pitch and roll estimation results for an inverted loop simulation in which fixed and random biases are added to all sensors (case 4). Error in pitch angle is of the order of $2 \mathrm{deg}$ (Fig. 3, left-bottom). Large roll errors (Fig. 4, right-top and right-bottom, $\approx 100 \mathrm{deg}$ ) occur only during the mathematical roll transition from 0 to $180 \mathrm{deg}$ or vice versa (around $\theta= \pm 90 \mathrm{deg}$ ). We have observed that this drastic increase in roll estimation error happens only for $|\theta|>80$ $\mathrm{deg}$, and the error is due to a difference in slope of roll transition (Fig. 4, top). Beyond $\theta=-80 \mathrm{deg}$, roll actually becomes undefined, and this transition is due to the quaternion formulation. There is no physical roll happening here.
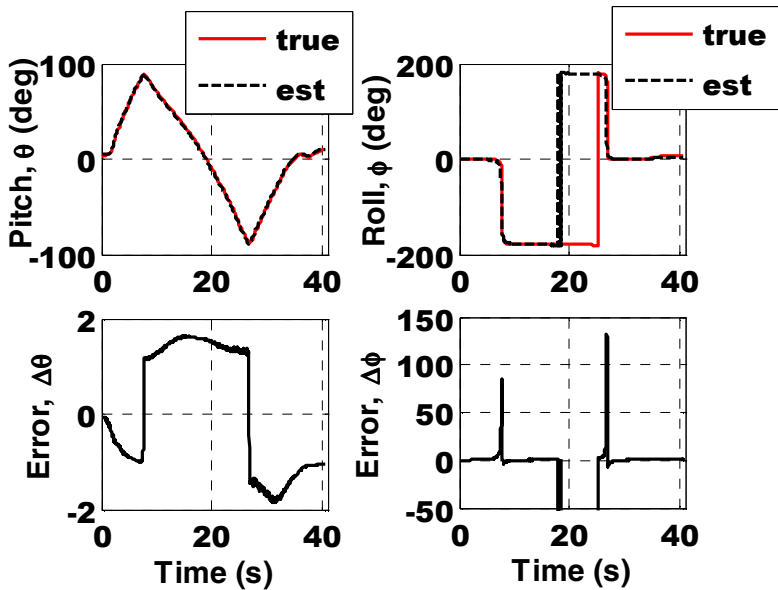

Fig. 3: Estimation results for an inverted-loop simulation with case 4 sensor noise and biases; pitch and roll angles.

Thus the only body rate information that the estimator receives is from pitch rate. Roll rate and yaw rate should be zero. But, when noise or biases are introduced in sensor data, it tends to make roll rate and yaw rate non-zero. Whatever information that the estimator has regarding roll or yaw is due to these sensor noise and biases. Hence the estimated transition flattens out (Fig. 4, top). Here we ignore the roll behaviour for $|\theta|>80$ deg based on the justification that, roll is anyway getting undefined (hence information not useful), and report the maximum estimation errors only for $|\theta| \leq 80$ deg (Table 2).

For the case given in Fig. 4, maximum roll estimation error for $|\theta| \leq 80 \mathrm{deg}$ is around $4 \mathrm{deg}$. Note that presence of wind turbulence or wind shear also affects the estimation results for an inverted loop in a similar manner (Table 2). This means the algorithm's inevitable approximation, by which wind speeds are ignored, and air-reference speeds are used as inertial speeds, degrades the estimation performance for an inverted loop performed under windy conditions. The maximum errors in pitch and roll for inverted-loop simulations are summarized in Table 2. Note that roll estimation error becomes more than $10 \mathrm{deg}$ only under severely-turbulent wind conditions.

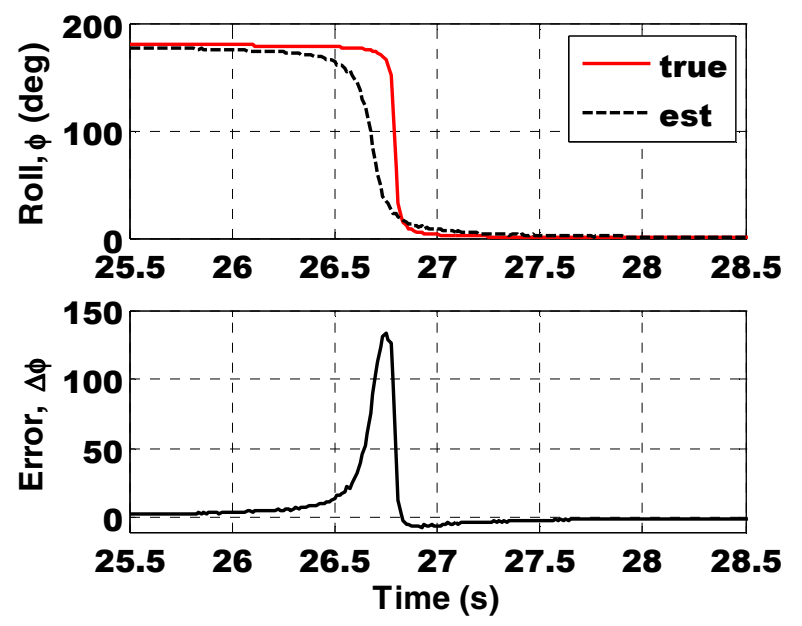

Fig. 4: Estimation results for an inverted-loop simulation with case 4 sensor noise and biases; a closer look at roll results around $\theta=-90 \mathrm{deg}$.

Table 2: Estimation errors (in deg) for Simulation II

\begin{tabular}{|l|c|c|}
\hline \multicolumn{1}{|c|}{ Simulation } & $\begin{array}{c}\text { Maximum roll } \\
\text { error } \\
(|\boldsymbol{\theta}| \leq \mathbf{8 0} \text { deg })\end{array}$ & $\begin{array}{c}\text { Maximum } \\
\text { pitch error }\end{array}$ \\
\hline CLEAN DATA & 0.0380 & 0.1261 \\
\hline CLEAN DATA + NOISE + BIAS & 3.4946 & 1.8596 \\
\hline $\begin{array}{l}\text { CLEAN DATA + SEVERE } \\
\text { TURBULENCE }\end{array}$ & 16.729 & 3.2778 \\
\hline $\begin{array}{l}\text { CLEAN DATA + NOISE + BIAS + } \\
\text { SEVERE TURBULENCE }\end{array}$ & 24.33 & 4.4868 \\
\hline CLEAN DATA + WIND SHEAR & 6.6202 & 1.1649 \\
\hline $\begin{array}{l}\text { CLEAN DATA + WIND SHEAR + } \\
\text { NOISE + BIAS }\end{array}$ & 7.1112 & 1.5361 \\
\hline
\end{tabular}

\subsection{Simulation III: full roll}

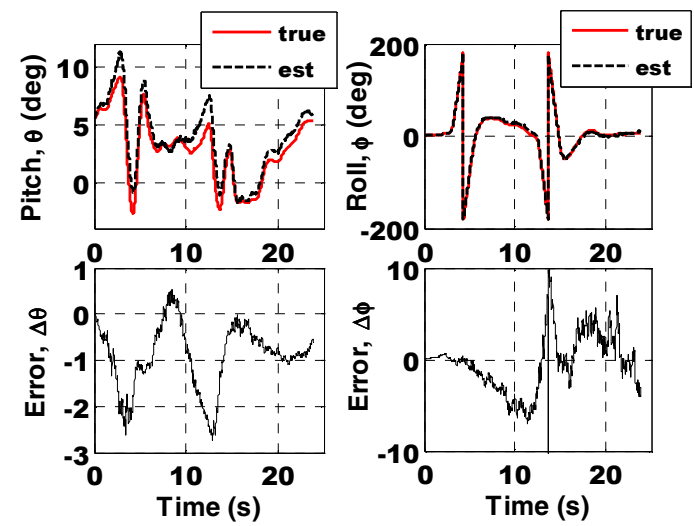

Fig. 5: Estimation results for a severe turbulence-affected full roll simulation with case 4 sensor noise and biases; pitch and roll angles. 
The pitch and roll estimation results shown in Fig. 5 is for a simulation containing a couple of full-roll manoeuvres performed under severe turbulence conditions. Also, fixed and random biases are introduced in all sensors, i.e. case 4 . In this case, maximum pitch and roll estimation errors are of the order of 3 and 10 degrees, respectively.

Estimation errors for full-roll maneuvers are much lesser than those for inverted loops, especially in roll angle. The reason for this is two-fold:

- In full rolls, aircraft is actually rolling and therefore estimator does receive a majority of reliable roll rate information, although it is corrupted with noise and bias. Whereas in the case of inverted loops, since there is no physical roll happening whatever information the estimator gets regarding roll is due to sensor noise and biases.

- The time scale of a roll transition during an inverted loop (within a second) is slightly faster than that of a full-roll maneuver $(\sim 2-3 \mathrm{sec})$. Hence, the slightest wind disturbance or sensor bias results in a larger error during the roll transition of an inverted loop.

Maximum estimation errors for different cases of full roll maneuvers are presented in Table 3. The performance is satisfactory.

Table 3: Estimation errors for Simulation III

\begin{tabular}{|l|c|c|}
\hline \multicolumn{1}{|c|}{ Simulation } & $\begin{array}{c}\text { Maximum roll } \\
\text { error }(\mathbf{d e g})\end{array}$ & $\begin{array}{c}\text { Maximum pitch } \\
\text { error (deg) }\end{array}$ \\
\hline CLEAN DATA & 1.2134 & 0.0669 \\
\hline $\begin{array}{l}\text { CLEAN DATA + NOISE + } \\
\text { BIAS }\end{array}$ & 4.7234 & 1.2302 \\
\hline $\begin{array}{l}\text { CLEAN DATA + SEVERE } \\
\text { TURBULENCE }\end{array}$ & 8.3604 & 1.7784 \\
\hline $\begin{array}{l}\text { CLEAN DATA + SEVERE } \\
\text { TURBULENCE + NOISE + } \\
\text { BIAS }\end{array}$ & 9.9753 & 2.7264 \\
\hline
\end{tabular}

Thus, results using simulation data show that estimation errors in pitch and roll remain within a maximum of 4.5 and 20 degrees, respectively. The larger errors occur only in the special case of an inverted loop in the presence of severe wind turbulence (Table 2). Excluding this severe turbulence case, errors in pitch and roll remain within 4 and 10 degrees, respectively.

\section{ESTIMATION RESULTS: FLIGHT DATA}

For majority of the 332 flights considered for evaluation, estimates were comparable with the data from GPS-assisted INS $(|\Delta \theta|<5 \mathrm{deg}$ and $|\Delta \phi|<10 \mathrm{deg})$. A select few cases such as full-roll, inverted-loop, and steep-climb/steep-dive manoeuvres which were producing relatively-large differences (only in roll) with respect to GPS-assisted INS are discussed in this section.

For most of the flight data evaluated, the three gyro biases are observed to settle down to specific constant values. These steady bias values are approximately constant for a particular aircraft.

\subsection{Flight data: inverted loop}

For inverted-loop maneuvers in actual flight, very similar to the results from simulation data (Figs. 3 and 4), the problematic region is around $\theta= \pm 90 \mathrm{deg}$ when roll flips from 0 to 180 or vice versa (Fig. 6). Various characteristics of the estimation error are the same as those for simulation data (no physical roll, roll getting undefined for $|\theta|>80 \mathrm{deg}$, roll transition due to quaternion formulation). Again, roll errors occur due to different transition slopes in estimated data and INS data (Fig. 7). We saw with simulation data that sensor biases and turbulent wind conditions can lead to such errors (Figs. 3 and 4). And since bias in $a_{y}$ and $a_{z}$ are not accounted for in the estimation algorithm, it is a potential cause for these errors. Maximum error in pitch angle is of the order of 2 deg (Fig. 6, bottom).

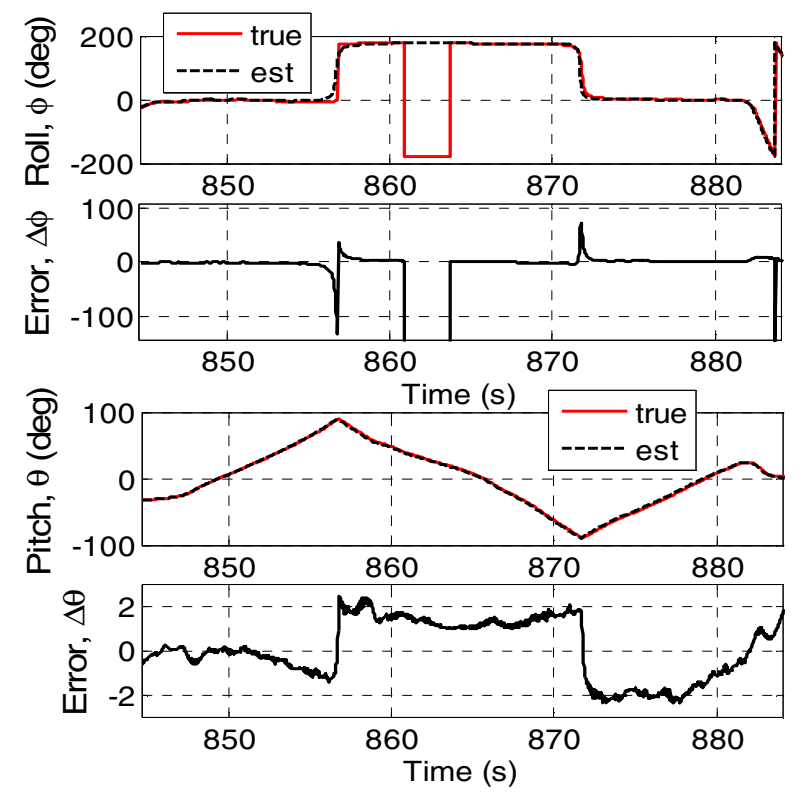

Fig. 6: Estimation results for a flight segment containing an inverted-loop manoeuvre; roll and pitch angles.

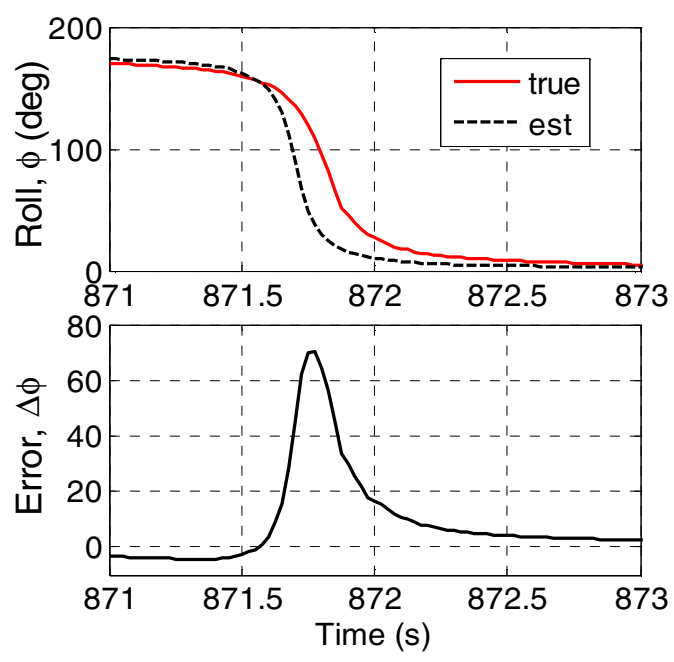

Fig. 7: Estimation results for a flight segment containing an inverted loop manoeuvre; a closer look at the roll results around $\theta=-90 \mathrm{deg}$. 
During inverted loops, in general, large roll errors occur only for pitch angles beyond $\pm 75 \mathrm{deg}$. For pitch angles, $|\theta| \leq 75$ deg, roll estimation errors, $|\Delta \phi| \leq 10 \mathrm{deg}$. It is in the pitch range $75<|\theta| \leq 80$ that, maximum roll errors increase to $\pm 20 \mathrm{deg}$. For $|\theta|>80$, roll errors are larger than $\pm 20 \mathrm{deg}$, which have been ignored (not documented) in the current evaluation. The justification is that beyond $\pm 80 \mathrm{deg}$ pitch, roll angle is not well-defined and hence its information is not useful. Moreover, physically, during an inverted loop, aircraft does not go through such a roll maneuver. Thus the conclusion is that, for inverted loops, roll estimation error using proposed estimation algorithm remains within \pm 20 deg for $|\theta| \leq 80 \mathrm{deg}$. Roll attitude information beyond $80 \mathrm{deg}$ pitch angle is not necessary for control law computations and loss of accuracy does not affect aircraft behaviour.

\subsection{Flight data: full roll}

During a full-roll manoeuvre, pitch estimation errors remain well within 5 deg (not shown). In Fig. 8, roll estimation errors during full rolls are seen to increase to around $15 \mathrm{deg}$. These are transition errors, i.e., relatively-large errors occur while the aircraft transitions from one roll position to another. Also, each full-roll maneuver is followed by a short-time drift error, and when the aircraft does a left roll and a right roll together, the drift errors cancel each other to make net zero error (Fig. 8, bottom).
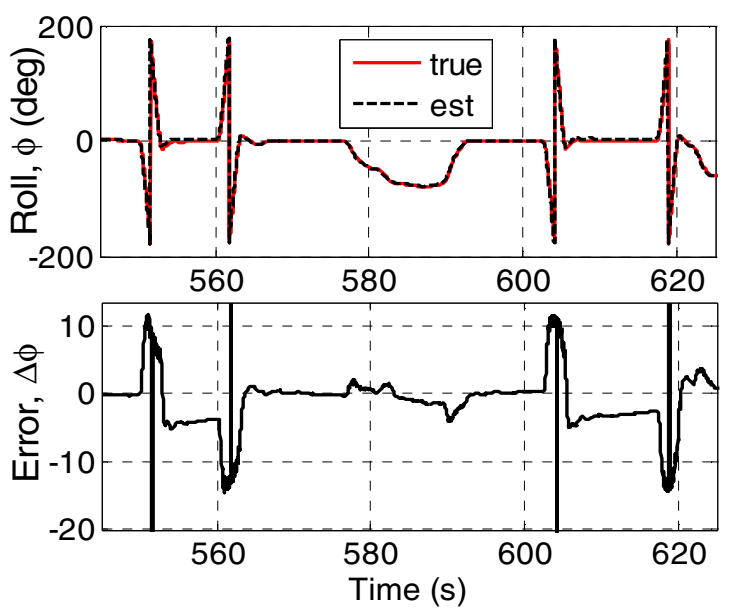

Fig. 8: Estimation results for a flight segment containing a series of full rolls; roll angle.

Note that, using flight data, there is an increase in roll estimation errors for full-rolls as compared to those obtained from using simulation data (Table 3). In general, estimation errors observed in results using flight data could be slightly higher than those got using simulation data (true data). This is because INS-GPS is estimating attitude data using information from its own sensors (hence, have its own estimation errors) which could have different noise and bias characteristics as compared to DFCC sensors, and we are comparing our estimation results (based on DFCC sensors only) with this data assuming that it is true attitude data.

\subsection{Flight data: steep climb and steep dive}

Figure 9 shows the estimation results for a flight segment containing a steep climb manoeuvre. The steep climb is performed at a pitch angle greater than 80 degrees. Estimation errors in pitch and flight path angle (not shown) remain within $2 \mathrm{deg}$ (Fig. 14, bottom). Large errors $(> \pm 20$ $\mathrm{deg}$ ) in roll estimation occur only for $\theta>80 \mathrm{deg}$. Similarly for steep dives, roll errors are large only for $\theta<-80 \mathrm{deg}$ (not shown). Thus, steep climbs and dives, in general, satisfy the condition that $|\Delta \phi| \leq 20 \mathrm{deg}$ for $|\theta| \leq 80 \mathrm{deg}$.
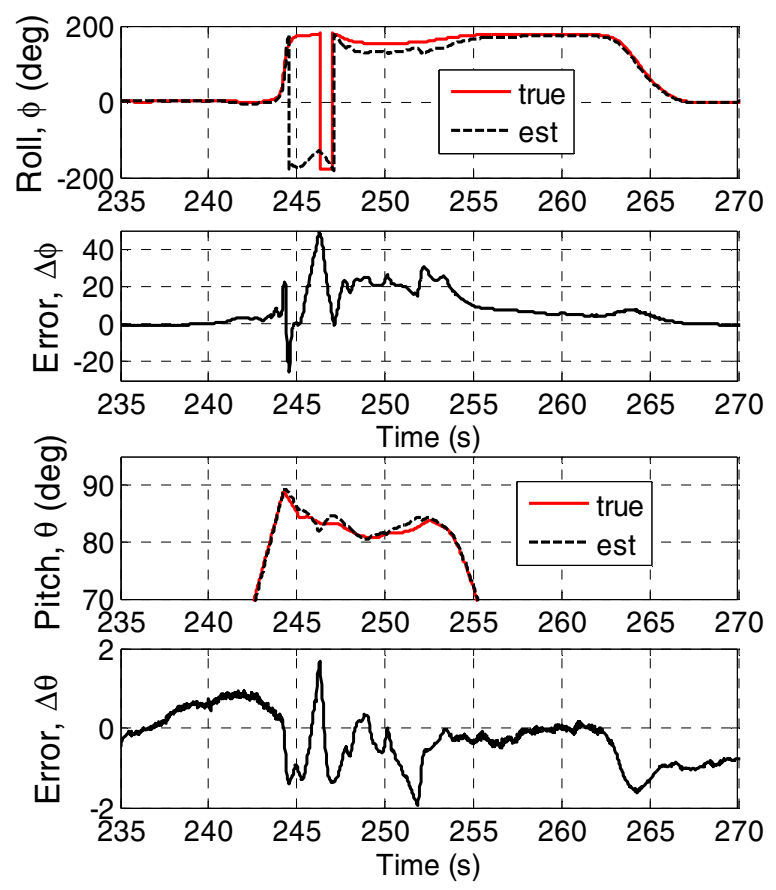

Fig. 9: Estimation results for a flight segment containing a steep climb; roll and pitch angles.

Thus, results from aircraft flight data show that estimation errors in pitch and roll stay within a maximum of 5 and 20 degrees, respectively. The larger errors are transient occurrences (not steady state) during transition period of full rolls (Fig. 8), during roll-flip $(|\theta|>75 \mathrm{deg})$ period of inverted loops (Fig. 6 and 7), and during steep climbs or dives when pitch angle crosses $|\theta| \approx 80 \mathrm{deg}$ (Fig. 9).

\section{ESTIMATION RESULTS: SUMMARY}

The maximum estimation errors observed in pitch and roll angles for different simulation and flight cases are summarized in Table 4. In the case of inverted-loop, steepclimb, and steep-dive manoeuvres, roll estimates are unreliable $(|\Delta \phi|>20 \mathrm{deg})$ for $|\theta|>80 \mathrm{deg}$. This information has been ignored in the current evaluation, since for this range of pitch angles, roll is not well-defined and hence its information is not useful.

In simulation results, absolute pitch errors are always less than $4.5 \mathrm{deg}$. Roll errors are larger than \pm 10 deg only in the special case of an inverted-loop manoeuvre under severe wind turbulence (Table 2). Excluding this unlikely case, roll estimation errors stay within $\pm 10 \mathrm{deg}$ for all other simulations. Thus, based on simulation data, we conclude 
that the algorithm achieves the target estimation accuracy of 5 and $10 \mathrm{deg}$ in pitch and roll, respectively.

Table 4: Final results

\begin{tabular}{|c|c|c|c|}
\hline & Cases & $\begin{array}{l}\text { Maximum } \\
|\Delta \phi| \text { (deg) }\end{array}$ & $\underset{(\text { deg })}{\operatorname{Maximum}}|\Delta \theta|$ \\
\hline \multirow{3}{*}{ } & $\begin{array}{c}\text { Inverted loop in the } \\
\text { presence of severe } \\
\text { turbulence }\end{array}$ & $\begin{array}{c}20 \\
(|\theta| \leq 80)\end{array}$ & 4.5 \\
\hline & Full roll (all cases) & 10 & 4 \\
\hline & $\begin{array}{l}\text { Rest of the cases } \\
\text { (inverted loop, } \\
\qquad|\theta| \leq 80)\end{array}$ & 10 & 4 \\
\hline \multirow{3}{*}{ 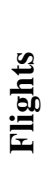 } & Full roll & 20 & 5 \\
\hline & $\begin{array}{l}\text { Inverted loop, steep } \\
\text { climb, steep dive }\end{array}$ & $\begin{array}{c}20 \\
(|\theta| \leq 80) \\
\end{array}$ & 5 \\
\hline & Rest of the cases & 10 & 5 \\
\hline
\end{tabular}

In the results using flight data, pitch estimation errors stay within $\pm 5 \mathrm{deg}$ for $97.9 \%$ of the 332 flights. The minority outlier flights $(=7)$, for which the maximum absolute pitch errors are between 5 and $6 \mathrm{deg}$, are omitted based on the following two additional reasons:

1) INS data itself is based on an estimator having its own errors, and hence need not be true,

2) There is a time synchronization mismatch between DFCC and INS data.

The same two reasons lead to larger roll estimation errors observed in flight data analysis (Table 4). Moreover, we saw that (Sections $5.1-5.3)$ the larger errors $(\Delta \phi \geq 10 \mathrm{deg}$ ) either transiently occur (not steady-state) during the transition period $(|\phi|>135 \mathrm{deg})$ of full rolls, or occur for $|\theta|>75$ deg, where roll is anyway getting undefined and hence its information is not useful. Thus, based on flight data too, we can conclude that the algorithm achieves the target estimation accuracy of 5 and $10 \mathrm{deg}$ in pitch and roll, respectively.

Also, the algorithm has been implemented and successfully run in a real-time distributed engineer-in-the-loop simulator, and thus, it meets the computational time constraints in closed-loop environment. As far as the processor is concerned, the requirement is a 32-bit processor which the Digital Flight Control Computer (DFCC) of the intended aircraft meets.

\section{CONCLUSIONS}

In the present work, performance of a novel attitude estimation algorithm has been evaluated using flight data from a high performance fighter aircraft and simulation data. For evaluation using flight data, 332 flights have been used. The correctness of estimation results is verified by comparing it with data from a GPS-assisted INS. In the case of simulation data, the simulation itself provides the true values. Based on the results obtained in the current work, the proposed estimation technique has been proven to perform with reasonable accuracy for flight control law applications.
Thus, this algorithm will be implemented in the DFCC of a high performance fighter aircraft for estimation of pitch and roll angles. These pitch and roll estimates will be used in the control laws to provide for gravity compensation during turn manoeuvres.

\section{ACKNOWLEDGMENTS}

The work is carried out under a project sanctioned by Aeronautical Development Agency (ADA), Bangalore. The authors sincerely thank Mr. Shyam Chetty, Director, NAL, Dr. Girija Gopalarathnam, Head FMCD, NAL, Dr. Girish Deodhare, TD IFCS, ADA, Dr. Vijay Patel, Scientist F, ADA, and Dr. A. A. Pashilkar, Head of Flight Simulation group, NAL for their valuable comments and encouragement.

\section{REFERENCES}

Crassidis, J.L., Markley, F.L., and Cheng, Y. (2007). A survey of nonlinear attitude estimation methods. AIAA Journal of Guidance, Control and Dynamics, Vol. 30 (1), 12-28.

Jung, D. and Tsiotras, P. (2007). Inertial attitude and position reference system development for a small UAV. AIAA Infotech at Aerospace, AIAA 2007-2763, May 7-10, CA, USA.

Kamali, C., Pashilkar, A.A., Deodhare, G., and Saraf, A. (2013). Attitude estimation of high performance aircraft. AIAA Guidance, Navigation and Control conference, AIAA 2013-4958, August 19-22, Boston, MA, USA.

Mahony, R., Hamel, T., and Pflimlin, J-M. (2008). Nonlinear complementary filters on the special orthogonal group. IEEE Transactions on Automatic Control, Vol. 53 (5), 1203-1217.

Park, S. and How, J., Examples of estimation filters from recent aircraft projects at MIT. MIT lecture notes, November 2004.

Titterton, D.H. and Weston, J.L. (2004). Strap-down Inertial Navigation Technology $-2^{\text {nd }}$ Edition. In N. Stewart and H. Griffiths (ed.), IEE Radar, Sonar, Navigation and Avionics Series 17. Institution of Electrical Engineers, Stevenage, UK.

Wendel, J. and Trommer, G.F. (2004). Tightly-coupled GPS/INS integration for missile applications. Journal of Aerospace Science and Technology, Vol. 8, 627-634. 